\title{
Feedback Error Compensation for Joint Adaptive Modulation and Diversity Combining*
}

\author{
Seyeong Choi \\ MCTR Lab. \\ LG Electronics \\ Anyang-shi, Kyungki-do, Korea \\ Email: seyeong.choi@gmail.com
}

\author{
Hong-Chuan Yang \\ Dept. of Electrical \& Computer Eng. \\ University of Victoria \\ BC, V8W 3P6, Canada \\ Email: hyang@ece.uvic.ca
}

\author{
Mohamed-Slim Alouini, Khalid A. Qaraqe \\ Dept. of Electrical Eng. \\ Texas A\&M University at Qatar \\ Education City, Doha, Qatar \\ Email: \{alouini, khalid.qaraqe\}@qatar.tamu.edu
}

\begin{abstract}
In this paper, we consider the effect of feedback error on the performance of the joint adaptive modulation and diversity combining (AMDC) scheme which was previously studied with an assumption of perfect feedback channels. In order to compensate for the performance degradation due to feedback error, we propose the path adjustment algorithm for diversity combining. We quantify the performance of the joint AMDC scheme in the presence of feedback error, in terms of the average spectral efficiency, the average number of combined paths, and the average bit error rate. Selected numerical examples are presented and discussed to illustrate the effectiveness of the proposed feedback error compensation strategy with adaptive combining.
\end{abstract}

Keywords - Adaptive modulation, diversity techniques, feedback error, and performance analysis.

\section{INTRODUCTION}

To meet the growing demand for a higher spectral efficiency as well as a good link reliability in wireless communications, many new technologies have been devised for the last few decades. Among them, adaptive modulation and diversity combining (AMDC) are known as important enabling techniques. Adaptive modulation [1], [2] aims to achieve high spectral efficiency while maintaining a certain target error rate performance, and diversity combining [3]-[5] can improve the link reliability of wireless fading channels by utilizing two or more communication channels with different characteristics.

Recently, as an attempt to obtain further improved spectral efficiency under the same error rate requirement, joint AMDC schemes were proposed and analyzed in [6] by employing more sophisticated diversity combining techniques such as generalized selection combining (GSC) [7], [8] and minimum selection-GSC (MS-GSC) [9], [10]. With the schemes in [6], the receiver jointly decides the proper modulation mode and diversity combiner structure based on the channel quality and the target error rate requirement. Different mode of operations have been considered based on the primary optimization criteria of the joint design. For example, the power-efficient (PE) AMDC scheme leads to a high processing power efficiency, the bandwidth-efficient (BE) AMDC scheme leads to a high

\footnotetext{
* This work was supported in part by the Qatar Foundation for Education, Science, and Community Development, Qatar, in part by Qatar Telecom (Qtel), Qatar, and in part by a Discovery Grant from NSERC, Canada.
}

bandwidth efficiency, and the bandwidth-efficient and powergreedy (BEPG) AMDC scheme leads to a high bandwidth efficiency as well as an improved power efficiency at the cost of a higher error rate in comparison with the BE AMDC scheme. For the analytical tractability, it is assumed in [6] that the feedback channel is error-free.

On the other hand, it is also very important to consider the impact of imperfect feedback channels, especially in the case that sufficient and powerful error control method cannot be implemented over the feedback channels. Note that feedback error may induce that the adaptive modulation mode actually used for transmission may be different from the true one selected by the receiver after adaptive combining. To accommodate for such scenarios and avoid the mode mismatch between the transmitter and the receiver, the transmitter needs to inform the receiver which mode it will be using before the actual data transmission. In the previous works [11], [12], the authors have proposed to utilize the adaptive receiver diversity to compensate for feedback error in AMDC systems with BEPG scheme. In particular, the receiver may combine more diversity paths, if possible, to compensate for the bit error rate (BER) degradation when the transmitter sends data with a higher modulation mode than the one selected by the receiver. On the other hand, the receiver may use less diversity paths (i.e., explore additional power savings) to save the receiver processing power when the transmitter sends data with a lower modulation mode.

In this paper, we complement the previous work by investigating two other AMDC schemes, namely $\mathrm{PE}$ and $\mathrm{BE}$ schemes. Similar to [11], [12], we investigate the impact of feedback error on these AMDC systems by analyzing their average number of combined paths, average spectral efficiency, and average BER performance. We also quantify the benefit that adaptive path adjustment will bring to these systems with imperfect feedback link. We show through selected numerical examples that the proposed compensation strategy can offer considerable error performance improvement with little loss in processing power and spectral efficiency in comparison with the no compensation case.

\section{Channel and System Model}

We follow exactly the same signal and channel model used in $[6$, Sec. II] which are omitted here for the brevity 
and due to the space limitations. Specifically, we adopt a block independent and identically distributed (i.i.d.) Rayleigh fading model where the fading coefficients corresponding to different diversity paths are constant through the data burst period. In addition, we assume either flat fading with multiple antennas or selective fading with RAKE receiver. With this assumption, different diversity paths correspond to different antennas or different resolvable paths. We consider a frequency division duplex (FDD) system where the frequency separation between uplink and downlink channels is much greater than the channel coherent bandwidth. As such, the forward and feedback channels experience independent fading.

Because of the imperfect feedback, the modulation mode used for transmission may be different from the one selected by the receiver. In particular, assume that after performing a series of operations based on PE or BE schemes [6, Secs. IIIA and IV-A], the receiver decides to use the modulation mode $j(0 \leq j \leq N)$ while combining $k(0 \leq k \leq L)$ strongest paths out of total $L$ available paths. The receiver sends the index of this modulation mode, $j$, to the transmitter via the feedback channel but due to a possible feedback error the transmitter may end up using mode $n(0 \leq n \leq N)$ instead of $j$ for transmission. We assume that the transmitter always informs the receiver about the actual transmitting mode ${ }^{1}$, i.e., the receiver has the knowledge of $n$ before the transmission occurs.

\section{PAth Adjustment FOR FEedback ERror COMPENSATION}

In this section, we present a detailed algorithm of the feedback error compensation for AMDC systems with PE and BE schemes. Note that while two transmit options ${ }^{2}$ in the case of poor channel quality were considered in [6], in this paper, for the sake of brevity, we confine our discussion to option 2 .

\section{A. PE Scheme}

Note that with PE scheme, in order to minimize the processing power, the receiver first tries to use the minimum number of strongest paths whose combined SNR is just above the threshold for binary phase-shift keying (BPSK), i.e., $\gamma_{T_{1}}$. After that, the receiver will find the highest supportable modulation mode while combining the same set of paths and feed the index of the chosen mode back. Depending of the actual mode used, the receiver may perform additional combiner update as follows. If the actual mode is lower than the mode selected by the receiver, i.e., $0<n \leq j$, no path update is required. In fact, just turning off the weakest path along will lead to unacceptable error performance for all $n>0$. On the other hand, if $j<n$, then the receiver may afford to combine

\footnotetext{
${ }^{1}$ In order to focus on the effect of feedback error, we assume that the actual mode notification is protected by the strong code.

${ }^{2}$ When the channel condition is very poor (i.e., the combined signal-tonoise ratio (SNR) of all available paths is below $\gamma_{T_{1}}$ where $\gamma_{T_{1}}$ is the lowest boundary threshold whose general form can be found in [6, Eq. (4)]), the transmitter with option 1 will always transmit with the lowest order transmission mode whereas the one with option 2 will stop transmission, buffer the data, and wait for more favorable channel conditions.
}

additional paths in order to make the combined SNR greater than the threshold, $\gamma_{T_{n}}$, and as such, satisfy the instantaneous BER performance requirement.

\section{B. BE Scheme}

With the BE scheme, in order to maximize the spectral efficiency, the receiver uses as many paths as possible to obtain the highest modulation mode by sequentially adding the strongest paths. If the highest mode is not supportable when all paths are combined, the receiver will decide the highest supportable mode and feedback its index. Hence, with BE scheme, when the chosen mode is less than $N$, i.e., $0<j<N$, all $L$ paths are always combined while less paths may be combined when $j=N$. As such, if the actual mode is lower than the selected mode, i.e., $0<n<j$, the receiver may reduce the number of combined paths by sequentially removing the weakest paths until either the combined SNR just satisfies the new output threshold, $\gamma_{T_{n}}$, or the number of combined paths is 1 . If $0<j \leq n$, then no path adjustment is necessary. Note that no transmission occurs when $n=0$.

\section{Quantification of Feedback Error}

To evaluate the effect of the feedback error and the compensation strategy, we need to determine the probability that the transmission mode $n$ is used while mode $j$ was selected by the receiver due to feedback error. We denote these mode transition probabilities by $q_{n, j}$ in the following. Note that these probabilities depend on the quality of the feedback channel and the signalling scheme used. In this work, we assume a PSK-based signalling scheme over the feedback channel. More specifically, we use $N+1$ different PSK symbols to represent the total $N+1$ transmission modes. The receiver needs to transmit only one symbol during the guard period to indicate the selected mode. If we assume that each transmission mode is sequentially mapped to each PSK symbol, then the decision region of the $i$ th symbol representing the $i$ th transmission mode will be the $i$ th wedge-shaped area in the PSK signal space. Therefore, the mode transition probability, $q_{n, j}$, is equal to the average probability that the decision variable for the feedback channel detection falls erroneously in the $n$th wedgeshaped area instead of the $j$ th wedge-shaped area. In [12, Table I], the transition probability, $q_{n, j}$, for this PSK-based sequential labeling scheme is calculated as a function of the average BER, $P_{b}$, and the required average symbol SNR, $\bar{\gamma}_{s}$, of the feedback channel when $N=3$.

\section{Performance Analysis in the Presence of FEEDBACK ERROR}

In this section, we study the performance of the PE and BE schemes with feedback error over i.i.d. Rayleigh fading channels. More specifically, we obtain its average spectral efficiency, average number of combined paths, and average BER whenever feasible in a simple fashion.

Keep in mind that only when there exists feedback error, the number of combined paths is changed by the proposed path adjustment algorithm in order to accommodate the erroneously 
selected modulation mode, $n$. Hence, the average spectral efficiency is just dependent on feedback error and is not affected by path adjustment. In case of no feedback error, the average spectral efficiency, $\eta$, of the adaptive system can be calculated as [1, Eq. (33)]

$$
\eta=\sum_{n=1}^{N} n \pi_{n},
$$

where $\pi_{n}$ is the probability that $n$th modulation mode is used. With feedback error, we can express the average spectral efficiency as

$$
\eta=\sum_{n=1}^{N} \sum_{j=0}^{N} n q_{n, j} \pi_{j}
$$

where $q_{n, j}$ is the mode transition probability due to feedback error. For the PE scheme, $\pi_{j}$ in (2) can be found in [6, Eq. (7)]. Since the spectral efficiency for the BE scheme is same as that for BEPG scheme, $\pi_{j}$ for the BE scheme can be found in [12, Eq. (15)].

The average BER for the adaptive modulation system can be calculated as [1, Eq. (35)]

$$
\mathrm{BER}=\frac{1}{\eta} \sum_{n=1}^{N} n \cdot \overline{\mathrm{BER}}_{n},
$$

where $\overline{\mathrm{BER}}_{n}$ is the average error rate when constellation size $n$ is used for transmission. When there is feedback error and the system does not adjust the number of combined paths, $\overline{\mathrm{BER}}_{n}$ can be calculated as

$$
\overline{\mathrm{BER}}_{n}=\sum_{j=0}^{N} q_{n, j} \int_{\gamma_{T_{j}}}^{\gamma_{T_{j+1}}} \operatorname{BER}_{n}(x) f_{\gamma}(x) d x
$$

where $f_{\gamma}(\cdot)$ is the probability density function (PDF) of the output SNR which has been obtained in [6, Eq. (6)] for the PE scheme and in [6, Eq. (13)] for the BE scheme. If the system adjusts the combiner structure for the case of feedback error, the calculation of $\overline{\mathrm{BER}}_{n}$ is more complicated since in this case the PDF of the output SNR is varying with combiner adjustment. In the following subsections, we consider the average BER and the average number of combined paths for each scheme with path adjustment.

\section{A. PE Scheme}

Assume that mode $n$ is used instead of $j$ due to feedback error. Let $f_{\gamma}^{-}(x)$ denote the PDF of the output SNR after adjustment for $j<n$ case and $f_{\gamma}^{+}(x)$ for $j \geq n$ case. Note that if $j<n$, the combined SNR after adjustment can be anywhere in the region of $\left[\gamma_{T_{j}}, \gamma_{T_{n+1}}\right]$ due to the effect of increasing the number of combined paths. On the other hand, if $j \geq n$, the combined SNR will be always in the region of $\left[\gamma_{T_{j}}, \gamma_{T_{j+1}}\right]$ as in this case, no path update is necessary based on the mode of operation of the PE scheme. Hence, $\overline{\mathrm{BER}}_{n}$ with path adjustment can be written as

$$
\begin{aligned}
\overline{\mathrm{BER}}_{n}= & \sum_{j=0}^{n-1} q_{n, j} \int_{\gamma_{T_{j}}}^{\gamma_{T_{n+1}}} \operatorname{BER}_{n}(x) f_{\gamma}^{-}(x) d x \\
& +\sum_{j=n}^{N} q_{n, j} \int_{\gamma_{T_{j}}}^{\gamma_{T_{j+1}}} \operatorname{BER}_{n}(x) f_{\gamma}^{+}(x) d x .
\end{aligned}
$$

Note that each integral limit in (5) is set to reflect in which interval the combined SNR after adjustment falls.

Let $l$ be the number of combined paths after path adjustment. Note that always $l \geq k$ for the PE scheme. If $j<n$, the receiver needs to combine more paths to raise the combined SNR above $\gamma_{T_{n}}$. In this case, $l>k$ if $k<L$, and $l=k$ if $k=L$. If $j \geq n$, then no branch update is necessary since the minimum number of paths, i.e., $k$ paths, are already combined. In this case, $l=k$. Hence, the cumulative distribution function (CDF), $F_{\gamma}^{-}(x)$, over $\left[\gamma_{T_{j}}, \gamma_{T_{n+1}}\right]$, after the update can be calculated for $j>0$ as

$$
\begin{aligned}
& F_{\gamma}^{-}(x) \\
& =\left\{\begin{array}{c}
\sum_{k=1}^{L} \operatorname{Pr}\left[\Gamma_{k-1: L}<\gamma_{T_{j}}<\Gamma_{k: L}<\gamma_{T_{j+1}},\right. \\
\left.\Gamma_{L: L}<x\right], \quad \gamma_{T_{j}}<x<\gamma_{T_{n}} ; \\
\sum_{k=1}^{L-1} \sum_{l=k+1}^{L} \operatorname{Pr}\left[\Gamma_{k-1: L}<\gamma_{T_{j}}<\Gamma_{k: L}<\gamma_{T_{j+1}},\right. \\
\left.\Gamma_{l-1: L}<\gamma_{T_{n}}<\Gamma_{l: L}<x\right], \quad \gamma_{T_{n}}<x<\gamma_{T_{n+1}}
\end{array}\right.
\end{aligned}
$$

and for $j=0$ as

$$
F_{\gamma}^{-}(x)= \begin{cases}\frac{\operatorname{Pr}\left[\Gamma_{L: L}<x\right]}{\operatorname{Pr}\left[\Gamma_{L: L}<\gamma_{T_{1}}\right]}, & 0<x<\gamma_{T_{1}} ; \\ 1, & \gamma_{T_{1}}<x,\end{cases}
$$

where $\Gamma_{i: j}$ is the sum of the $i$ largest SNRs among $j$ ones, i.e., $\Gamma_{i: j}=\sum_{k=1}^{i} \gamma_{k: j}$ where $\gamma_{k: j}$ is the $k$ th order statistics among $j$ ones (see [8] for terminology)). Based on the mode of operation of the PE scheme, the CDF, $F_{\gamma}^{+}(x)$, over $\left[\gamma_{T_{j}}, \gamma_{T_{j+1}}\right]$ is just the CDF of the combined SNR with MS-GSC which can be written as

$$
F_{\gamma}^{+}(x)=\sum_{k=1}^{L} \operatorname{Pr}\left[\Gamma_{k-1: L}<\gamma_{T_{j}}<\Gamma_{k: L}<x\right],
$$

where we define $\Gamma_{0: L}=0$. After differentiating (6), (7), and (8) with respect to $x$, we can finally obtain $f_{\gamma}^{-}(x)$ and $f_{\gamma}^{+}(x)$ in (5). The average number of combined paths also can be obtained by deriving the distribution of the actual number of combined paths, $l$, from (6). Unfortunately, solving (6) in closed-form is quite complicated since it requires us to use 4-dimensional joint PDF of order statistics. Hence, we rely on the computer simulation for (6).

\section{B. BE Scheme}

The average number of combined paths, $\bar{N}_{c}$, of the $\mathrm{BE}$ scheme without feedback error compensation can be found in [6, Eq. (14)]. With path adjustment, as mentioned in Section III-B, if $0<n<j$, the receiver reduces the combined paths while if $0<j \leq n$, then no path adjustment is required. If we let $P_{l, n, j}$ denote the probability that mode $n$ is used with $l$ combined paths after path adjustment while the true 
constellation size is $j$, then the average number of combined paths with feedback error is given by

$$
\begin{aligned}
\bar{N}_{c} & =\sum_{l=1}^{L} l\left(\sum_{n=1}^{N-1} \sum_{j=n+1}^{N} q_{n, j} P_{l, n, j}+q_{N, N} P_{l, N, N}\right) \\
& +\sum_{j=0}^{N-1} \sum_{n=j}^{N} q_{n, j} \operatorname{Pr}\left[\gamma_{T_{j}}<\Gamma_{L: L}<\gamma_{T_{j+1}}\right] \times \begin{cases}L, & n \neq 0 \\
0, & n=0 .\end{cases}
\end{aligned}
$$

Note that $P_{l, n, j}$ is valid only when $n<j \leq N$ and $n=j=$ $N$, and can be found in [12, Eqs. (11)(12)(13)].

Similarly, for the average BER, we consider two cases separately. Let $f_{\gamma}^{-}(x)$ denote the PDF of the output SNR in case that always $L$ paths are combined, and $f_{\gamma}^{+}(x)$ in case that $l(0<l \leq L)$ paths are required after path adjustment. Based on the path adjustment algorithm, always $L$ paths are combined when $j \leq n$ if $n<N$ and $j<n$ if $n=N$. Therefore, $\overline{\mathrm{BER}}_{n}$ can be written as

$$
\begin{aligned}
& \overline{\mathrm{BER}}_{n} \\
& =\left\{\begin{array}{l}
\sum_{j=0}^{n} q_{n, j} \int_{\gamma_{T_{j}}}^{\gamma_{T_{j+1}}} \operatorname{BER}_{n}(x) f_{\gamma}^{-}(x) d x \\
+\sum_{j=n+1}^{N} q_{n, j} \int_{\gamma_{T_{n}}}^{\gamma_{T_{n+1}}} \operatorname{BER}_{n}(x) f_{\gamma}^{+}(x) d \gamma, \quad n<N ; \\
\sum_{j=0}^{n-1} q_{n, j} \int_{\gamma_{T_{j}}}^{\gamma_{T_{j}}} \operatorname{BER}_{n}(x) f_{\gamma}^{-}(x) d x \\
+\sum_{j=n}^{N} q_{n, j} \int_{\gamma_{T_{n}}}^{\gamma_{T_{n+1}}} \mathrm{BER}_{n}(x) f_{\gamma}^{+}(x) d \gamma, \quad n=N,
\end{array}\right.
\end{aligned}
$$

where $f_{\gamma}^{-}(x)$ is simply the PDF of the $L / L$-GSC output SNR [5, Eq. (9.433)] and $f_{\gamma}^{+}(x)$ over the range of $\left[\gamma_{T_{n}}, \gamma_{T_{n+1}}\right]$ can be found in [12, Eq. (20)]. Substituting (10) into (3) leads to the desired average BER of the BE scheme with feedback error.

\section{NUMERICAL EXAMPLES}

In this section, we illustrate the simulation and analytical results derived in the previous section with some selected numerical examples. Note that we relied on simulation results only for the average number of combined paths and the average BER of the PE scheme with path adjustment while we obtained the exact analytical results for all other performance measures. In what follows, we use the transition probability, $q_{n, j}$, in Table [12, Table I] obtained from the labeling scheme presented in Section III-C.

Fig. 1 shows the average spectral efficiency of the three schemes for different average BERs, $P_{b}$, of the feedback channel as a function of the average SNR per path, $\bar{\gamma}$, when $L=5, N=3$, and $\mathrm{BER}_{0}=10^{-3}$. While being almost the same as that in the case of no feedback error when $P_{b}=10^{-2}$ and $10^{-3}$, the average spectral efficiency with $P_{b}=10^{-1}$ is larger over the low SNR region and smaller over the high SNR region than that without feedback error. Note that if the larger modulation mode $(N>3)$ is used, the difference in the average spectral efficiency between the cases of feedback error and no feedback error will be more distinct.

In Fig. 2, we present the average number of combined paths with the same parameters used in Fig. 1. From this figure, we can observe that if the average BER of the feedback channel

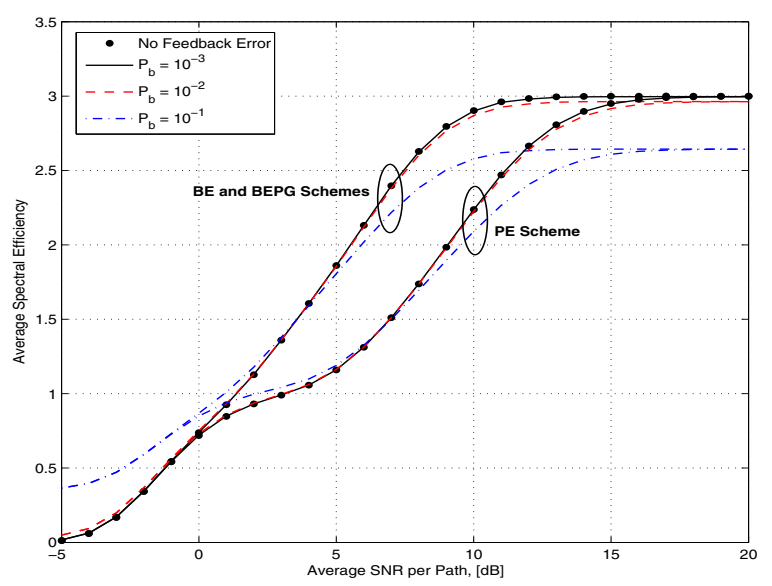

Fig. 1. Average spectral efficiency for different values of average BER, $P_{b}$, of the feedback channel as a function of the average SNR per path, $\bar{\gamma}$, when $L=5, N=3$, and $\mathrm{BER}_{0}=10^{-3}$.

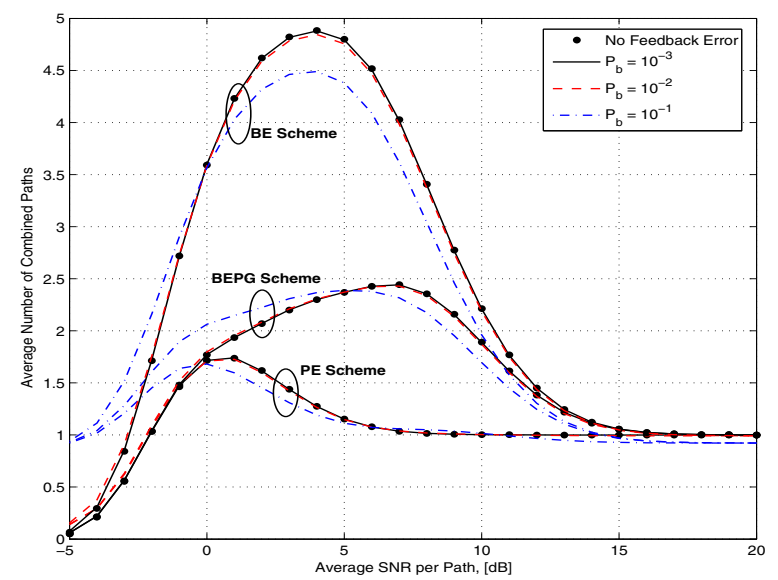

Fig. 2. Average number of combined paths for different values of average BER, $P_{b}$, of the feedback channel as a function of the average SNR per path, $\bar{\gamma}$, when $L=5, N=3$, and $\mathrm{BER}_{0}=10^{-3}$.

is relatively small, i.e., $P_{b}=10^{-2}$ and $10^{-3}$, the average number of combined paths is almost the same as that in the case of no feedback error, which means in this case feedback error does not significantly affect this performance measure. On the other hand, when $P_{b}=10^{-1}$, we can see that the system requires more paths over the low SNR region and less paths over the high SNR region. Note that as the average BER of the feedback channel is increasing, the possibility of path adjustment is also increasing because of the high chance of having different values between $n$ and $j$. In this case, for the low SNR region, the receiver has to combine more paths in order to satisfy the actual boundary threshold, $\gamma_{T_{n}}$, which is possibly larger than the true threshold, $\gamma_{T_{j}}$, while for the high SNR region, less paths are required to meet $\gamma_{T_{n}}$ which is possibly smaller than $\gamma_{T_{j}}$.

Finally, we examine the BER performance in Figs. 3 and 


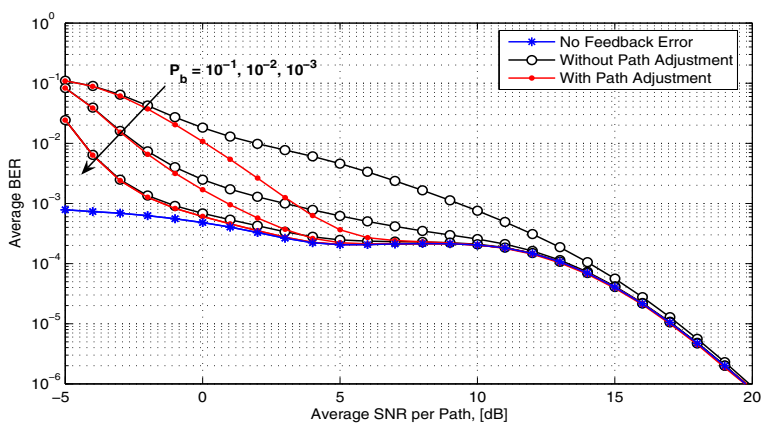

(a) PE scheme

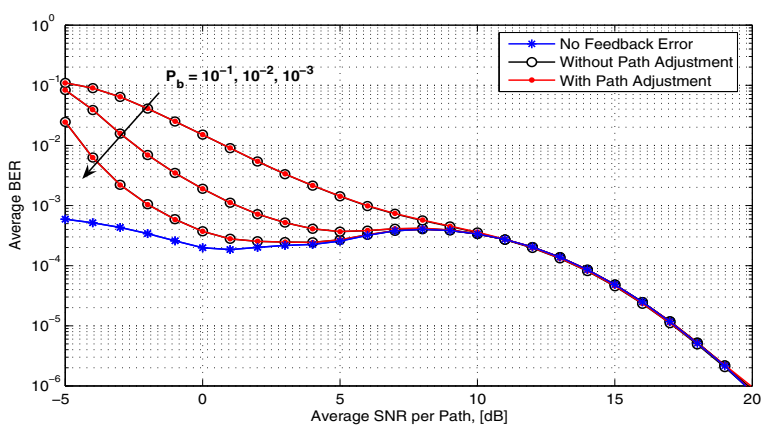

(b) BE scheme

Fig. 3. Average BER of (a) PE and (b) BE schemes for different values of average BER, $P_{b}$, of the feedback channel as a function of the average SNR per path, $\bar{\gamma}$, when $L=5, N=3$, and $\mathrm{BER}_{0}=10^{-3}$.

4. Fig. 3 shows that feedback error considerably degrades the BER performance especially for the low SNR region. It is very interesting to see that path adjustment for the BE scheme does not improve the BER performance while it affects considerably the BER performance of the PE scheme. Note that for the PE scheme, path adjustment helps improve the performance as the receiver may need additional paths when a higher mode $(n>j)$ is selected due to feedback error. For the BE scheme, all $L$ paths will be combined for $j<N$. Hence, even if $n>j$, the receiver can not combine more paths and as such, the error performance suffers in the same way as no path adjustment case. Note that the poor error performance due to feedback error (without path adjustment) is mainly due to the case where a higher mode is actually used. On the other hand, path adjustment for the BE scheme helps save some path combining, as can be seen in Fig. 2 . Note that over the 5-10 dB SNR region, the average number of combined path of the PE scheme slightly increases when there are feedback errors whereas that for the BE scheme decreases considerably. The effectiveness of path adjustment for the PE scheme in comparison with other schemes can be more clearly seen from Fig. 3. From this figure, we can observe that the proposed compensation strategy can offer considerable error performance improvement for the PE scheme in comparison with other schemes with little loss in processing power and spectral efficiency.

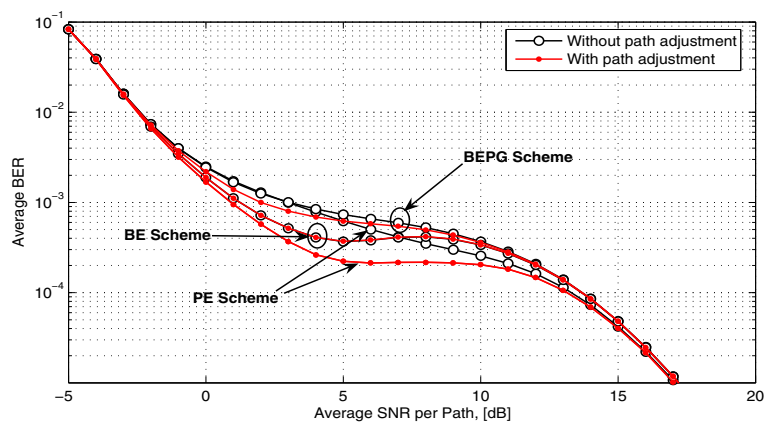

(a) $P_{b}=10^{-2}$

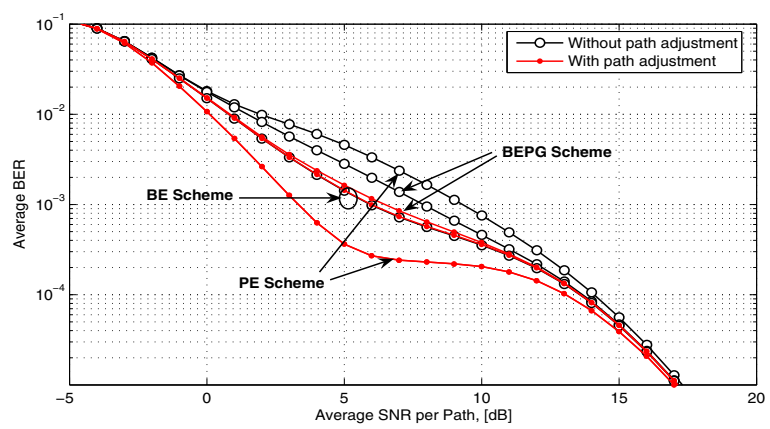

(b) $P_{b}=10^{-1}$

Fig. 4. Average BER of the three schemes for (a) $P_{b}=10^{-2}$ and (b) $P_{b}=$ $10^{-1}$ as a function of the average SNR per path, $\bar{\gamma}$, when $L=5, N=3$, and $\mathrm{BER}_{0}=10^{-3}$.

\section{REFERENCES}

[1] M.-S. Alouini and A. J. Goldsmith, "Adaptive modulation over Nakagami fading channels," Kluwer J. Wireless Commun., vol. 13, no. 1, pp. 119-143, May 2000.

[2] K. J. Hole, H. Holm, and G. E. Øien, "Adaptive multidimentional coded over flat fading channels," IEEE J. Select. Areas Commun., vol. 18, no. 7, pp. 1153-1158, Jul. 2000.

[3] N. C. Beaulieu, "Introduction to "linear diversity combining techniques"," Proc. IEEE, vol. 91, no. 2, pp. 328-356, Feb. 2003.

[4] G. L. Stüber, Principles of Mobile Communication, 2nd ed. Norwell, MA: Kluwer Academic Publishers, 2001.

[5] M. K. Simon and M.-S. Alouini, Digital Communication over Fading Channels, 2nd ed. New York, NY: John Wiley \& Sons, 2005.

[6] H.-C. Yang, N. Belhaj, and M.-S. Alouini, "Performance analysis of joint adaptive modulation and diversity combining over fading channels," IEEE Trans. Commun., vol. 55, no. 3, pp. 520-528, Mar. 2007.

[7] T. Eng, N. Kong, and L. B. Milstein, "Comparison of diversity combining techniques for Rayleigh-fading channels," IEEE Trans. Commun., vol. 44, no. 9, pp. 1117-1129, Sep. 1996.

[8] M.-S. Alouini and M. K. Simon, "An MGF-based performance analysis of generalized selection combining over Rayleigh fading channels," IEEE Trans. Commun., vol. 48, no. 3, pp. 401-415, Mar. 2000.

[9] R. K. Mallik, P. Gupta, and Q. T. Zhang, "Minimum selection GSC in independent Rayleigh fading," IEEE Trans. Veh. Technol., vol. 54, no. 3, pp. 1013-1021, May 2005.

[10] H.-C. Yang, "New results on ordered statistics and analysis of minimumselection generalized selection combining (GSC)," IEEE Trans. Wireless Commun., vol. 5, no. 7, pp. 1876-1885, Jul. 2006.

[11] S. Choi, M.-S. Alouini, K. A. Qaraqe, and H.-C. Yang, "Joint adaptive modulation and diversity combining with feedback error compensation," in Proc. IEEE Veh. Technol. Conf. (VTC'08), Calgary, Canada, Sep. 2008.

[12] S. Choi, H.-C. Yang, M.-S. Alouini, and K. A. Qaraqe, "Joint adaptive modulation and diversity combining with feedback error compensation," University of Victoria, BC, Canada, Tech. Rep. Available at http://hdl.handle.net/1828/1053, Aug. 2008. 